\title{
Status of the PALM-3000 high-order adaptive optics system
}

\section{Antonin Bouchez, Richard Dekany, John Angione, Christoph Baranec, Khanh Bui, et al.}

Antonin H. Bouchez, Richard G. Dekany, John R. Angione, Christoph Baranec, Khanh Bui, Rick S. Burruss, Justin R. Crepp, Ernest E. Croner, John L. Cromer, Stephen R. Guiwits, David D. S. Hale, John R. Henning, Dean Palmer, Jennifer E. Roberts, Mitchell Troy, Tuan N. Truong, Jeffry Zolkower, "Status of the PALM-3000 high-order adaptive optics system," Proc. SPIE 7439, Astronomical and Space Optical Systems, 74390H (17 September 2009); doi: 10.1117/12.826571

Event: SPIE Optical Engineering + Applications, 2009, San Diego, California, United States 


\title{
Status of the PALM-3000 high-order adaptive optics system
}

\author{
Antonin H. Bouchez ${ }^{*}$, Richard G. Dekany ${ }^{a}$, John R. Angione ${ }^{b}$, Christoph Baranec ${ }^{a}$, \\ Khanh Bui $^{a}$, Rick S. Burruss ${ }^{b}$, Justin R. Crepp ${ }^{a}$, Ernest E. Croner ${ }^{a}$, John L. Cromer ${ }^{a}$, \\ Stephen R. Guiwits ${ }^{b}$, David D.S. Hale ${ }^{a}$, John R. Henning ${ }^{c}$, Dean Palmer ${ }^{b}$, \\ Jennifer E. Roberts ${ }^{b}$, Mitchell Troy ${ }^{b}$, Tuan N. Truong ${ }^{b}$, Jeffry Zolkower ${ }^{c}$ \\ ${ }^{a}$ Caltech Optical Observatories, California Institute of Technology, Pasadena, CA, USA 91125; \\ ${ }^{b}$ Jet Propulsion Laboratory, California Institute of Technology, Pasadena, CA, USA 91109; \\ ${ }^{c}$ Palomar Observatory, California Institute of Technology, Palomar Mountain, CA, USA 92060
}

\begin{abstract}
The PALM-3000 upgrade to the Palomar Adaptive Optics system on the 5.1 meter Hale telescope will deliver extreme adaptive optics correction in near-infrared wavelengths and diffraction-limited images in visible wavelengths. PALM3000 will use a 3388-actuator tweeter and a 241 -actuator woofer deformable mirror, a Shack-Hartmann wavefront sensor with selectable pupil sampling, and an innovative wavefront control computer based on a cluster of 17 graphics processing units to correct wavefront aberrations at scales as fine as $8.1 \mathrm{~cm}$ at the telescope pupil using natural guide stars. The system is currently undergoing integration and testing, with deployment at Palomar Observatory planned in early 2011. We present the detailed design of key aspects of the adaptive optics system, and the current status of the deformable mirror characterization, wavefront sensor performance, and testbed activities.
\end{abstract}

Keywords: Extreme Adaptive Optics, Deformable Mirrors, Wavefront Sensing

\section{INTRODUCTION}

Since the discovery of the first substellar object at Palomar Observatory in $1995^{1}$, improvements in adaptive optics (AO) performance have gradually driven the detection limit of such objects down into the planetary regime. In the last four years, several extrasolar planet candidates have been directly imaged using VLT, Keck, and Gemini AO systems $^{2,3,4}$. All of these detections were at separations greater than 0.5 arcsec around particularly young stars $(<100$ $\mathrm{Ma}$ ), and with contrast ratios less than $10^{4}: 1$. In contrast to planets identified by other techniques, those directly imaged using adaptive optics offer the exciting possibility of the detailed study of their physical properties and even atmospheric composition.

With a predicted RMS residual wavefront error of $94 \mathrm{~nm}$ in median atmospheric conditions - equivalent to a Strehl ratio of $88 \%$ in $\mathrm{H}$ band $(\lambda=1.64 \mu \mathrm{m})$ - the PALM-3000 AO system is specifically designed to detect and characterize extrasolar planets. Using the Project $1640^{5}$ coronagraphic integral field spectrograph (IFS) built by the American Museum of Natural History and a post-coronagraphic calibration wavefront sensor contributed by the NASA Jet Propulsions Laboratory, we expect to reach contrast levels of greater than $10^{6}: 1$ at 0.5 arcseconds from bright stars in the raw data. Post-processing techniques which distinguish quasi-static atmospheric and instrumental speckles from astrophysical sources based on their spectral signature should extend companion detectability nearly another order of magnitude $^{6}$. This will bring the super-Jupiters of older stellar systems, and lower mass planets (approaching $1 \mathrm{M}_{\mathrm{J}}$ ) in young systems, within reach of observation. Most importantly, the IFS will gather low-resolution spectra of each candidate, addressing such questions as the temperature and atmospheric composition of these alien worlds.

Compared to planned high-contrast AO systems on larger diameter telescopes ${ }^{7,8}$, PALM-3000 benefits from a higher spatial bandwidth of correction and a larger control radius. Although high-contrast detection capability theoretically scales as $\mathrm{D}^{4} / \sigma^{2}$, where $\mathrm{D}$ is the telescope diameter and $\sigma$ is the residual RMS wavefront error, systematic errors actually limit all existing high-contrast instruments. By controlling high spatial frequency systematic errors, the current Palomar AO system already obtains contrast comparable to the best ground-based measurements on larger telescopes ${ }^{9}$. The high actuator density and smaller aperture additionally leads to a larger control radius $N \lambda / 2 \mathrm{D}$, where $N$ is the number of

* Send correspondence to A.H.B.: E-mail: abouchez@astro.caltech.edu, Telephone: 6263958915.

Astronomical and Space Optical Systems, edited by Penny G. Warren, Cheryl J. Marshall, Robert K. Tyson, Michael Lloyd-Hart, James B. Heaney, E. Todd Kvamme, Proc. of SPIE Vol. 7439, 74390H · C 2009 SPIE · CCC code: 0277-786X/09/\$18 · doi: 10.1117/12.826571 
actuators across the pupil. We therefore expect the "dark hole" of PALM-3000 to extend to 2.1 arcseconds radius at $\lambda=1.64 \mu \mathrm{m}$, leading to a potential discovery volume many times larger than that of other planet-finding instruments.

Two additional instruments, each addressing different scientific goals and already in use with the PALMAO system, round out the PALM-3000 suite. The PHARO infrared camera and spectrograph ${ }^{10}$ is the current workhorse instrument of the PALMAO system, providing imaging and grism spectroscopy at near-infrared wavelengths over a 25-40 arcsecond field. With great flexibility and only $35 \mathrm{~nm}$ of internal wavefront error, PHARO is routinely used to test novel coronagraphic techniques ${ }^{11}$ and is well suited for use with PALM-3000. The SWIFT I-z band AO-assisted integral field spectrograph $^{12}$ is designed to complement di $\bullet$ raction-limited near-infrared IFS on $8-10 \mathrm{~m}$ class telescopes in resolution and sensitivity by combining high order AO correction, low sky background, and sensitive deep depletion CCDs. It will benefit from the greatly improved correction in the visible provided by PALM-3000.

The PALM-3000 project is an upgrade to the successful PALMAO system ${ }^{13}$ currently in service at the Cassegrain focus of the 5.1 meter Hale Telescope at Palomar Observatory. It will reuse many of the components of the current system, including the optical bench and telescope mounting interface, handling fixtures, science instruments, 241actuator deformable mirror (LODM), and most large optics. Added to these will be a state-of-the-art 3388-actuator tweeter deformable mirror (HODM), a wavefront sensor with up to $63 \times 63$ sampling in the pupil plane, and a new wavefront reconstructor computer. The preliminary design of some of these components has been described previously ${ }^{14,15,16}$. This paper presents the detailed design of all major subsystems, and provides an update on the status of integration and testing.

\section{OPTICAL RELAY}

The design of the PALM-3000 optical relay was strongly constrained by the necessity to reuse the optical bench and offaxis parabolas, and preserve the location of the science focus. The final design is illustrated in Figure 1. The optical bench will be mounted parallel to the Cassegrain mounting flange, with alignment and calibration sources located in the intervening space. The telescope beam passes through a hole in the bench to the relay optics mounted on the far side.

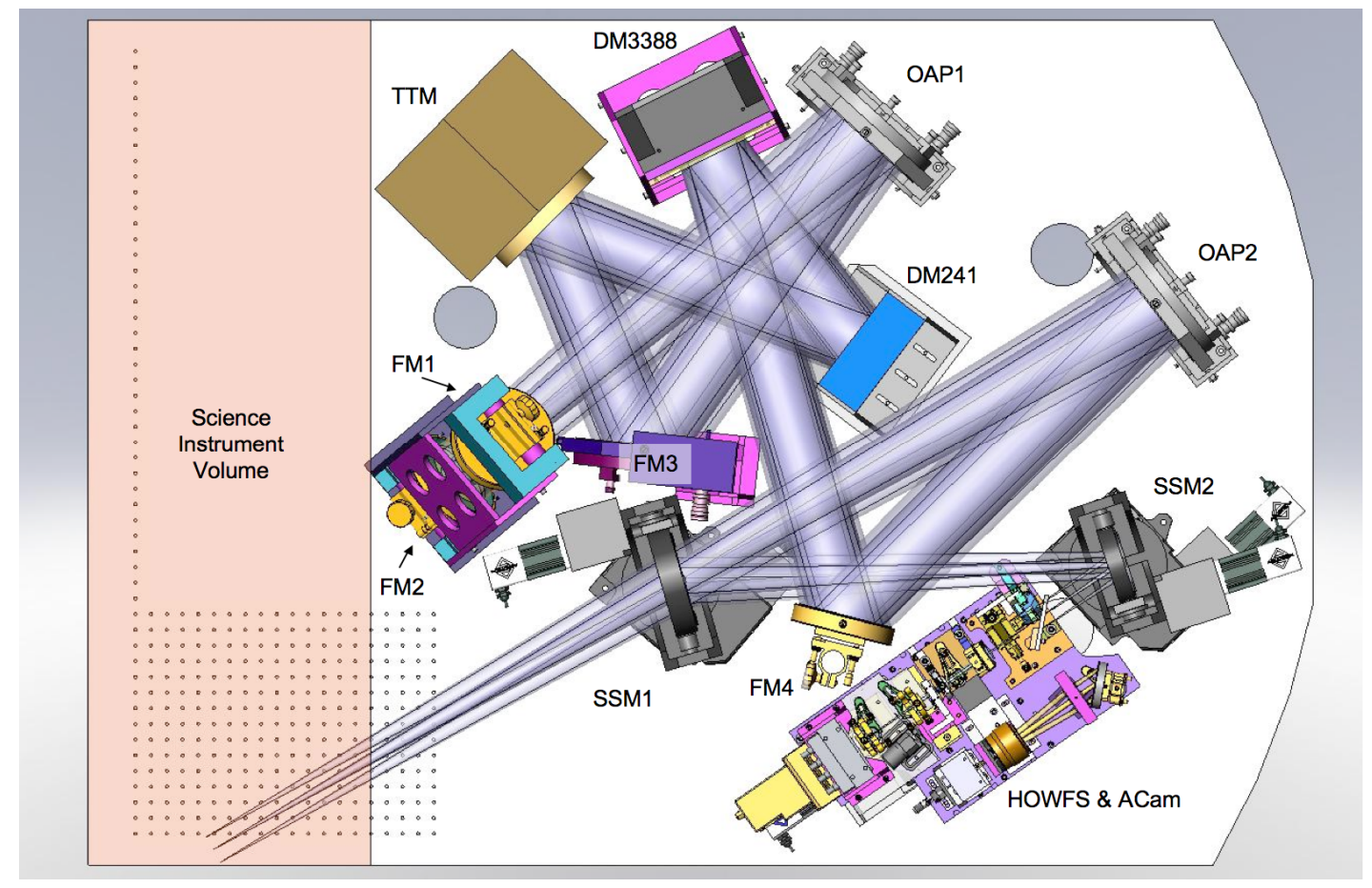

Figure 1. Rendering of the PALM-3000 optical bench showing a 120 arcsecond diameter beam. The optics illustrated here hang below the bench when installed at the Cassegrain focus of the Hale telescope. 

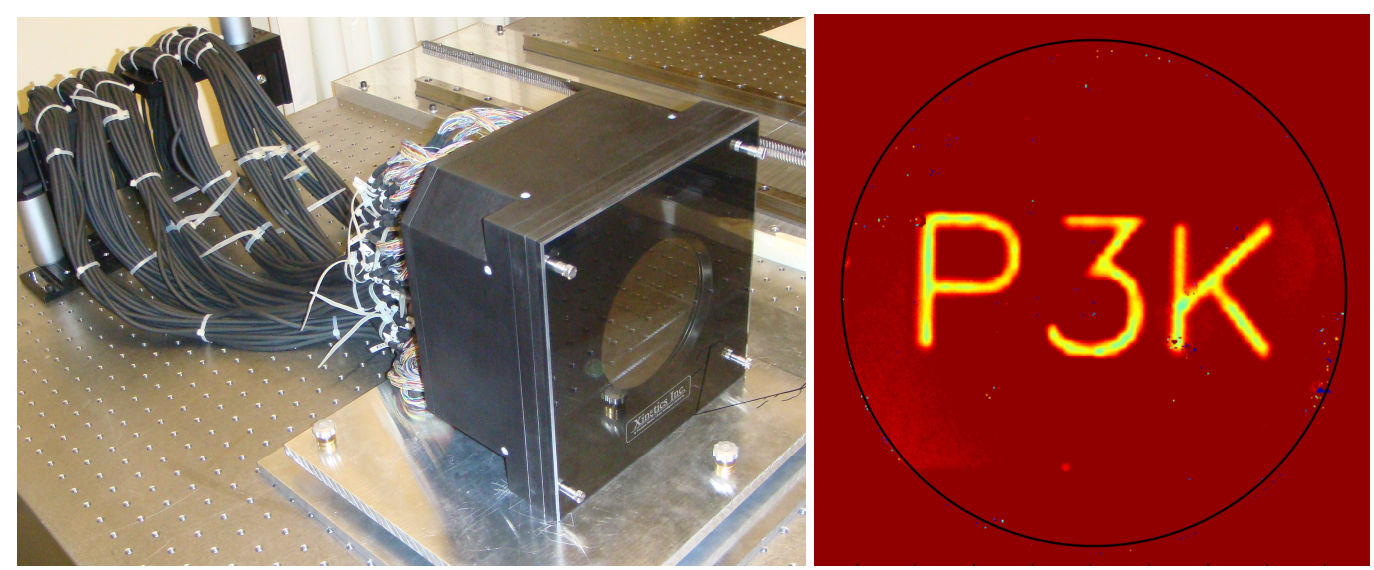

Figure 2. Left: The 3388-actuator deformable mirror cabled for optical testing in the laboratory at Xinetics Inc. Right: Zygo interferometer surface map of the deformable mirror with actuators spelling "P3K". The surface actuation is $\sim 100 \mathrm{~nm}$

Two flats in a "figure 4" (FM1 \& FM2) fold the beam into the relay. The relay consists of an off-axis parabola (OAP1), a fold flat (FM3), tip/tilt mirror (TTM), woofer DM (DM241), tweeter DM (DM3388), fold flat (FM4), and the second off-axis parabola (OAP2). A manually selectable dichroic in a gimbal mount (SSM1) reflects shorter wavelengths towards a second gimbaled mirror (SSM2) and the wavefront sensor and acquisition camera (HOWFS, ACam). Dichroics with cutoff wavelengths of $\lambda=650,750,960$, and $1050 \mathrm{~nm}$, and a 50/50 beamsplitter, are available for use with the various science instruments.

The optical relay passes an unvignetted 120 arcsecond diameter field to the science focus. This wide field was preserved to allow the future addition of laser guidestar capability. With on-axis static aberrations corrected by the deformable mirrors (primarily astigmatism due to the tilted dichroic, and tolerance for alignment error), field aberrations at the extreme corners of the largest science field, 30 arcseconds off axis, will suffer just $32 \mathrm{~nm}$ of RMS wavefront error.

All reflective relay optics will be coated with protected silver. Including telescope optics and a $1 \%$ loss at each surface due to coating degradation over time, we expect the average transmission to the science focus over $\lambda=1.1-2.3 \mu \mathrm{m}$ to be $65 \%$ when using the $\lambda=960 \mathrm{~nm}$ cutoff dichroic. The transmission to the wavefront sensor will be $58 \%$ over $\lambda=0.5$ $0.9 \mu \mathrm{m}$ due to the lower average reflectivity of the aluminum-coated telescope mirrors in the visible.

The design of the optical relay mechanics is driven by alignment tolerances in both the image and pupil planes, in the constantly changing gravity environment of the Cassegrain focus. Both the PHARO and Project 1640 instruments require image stability of 5 milliarcseconds (mas) peak-to-peak over a $300 \mathrm{~s}$ exposure to limit long-exposure Strehl degradation to $<5 \%$ in $\mathrm{H}$ band. We have already demonstrated active flexure compensation to this level during long exposures using the PALMAO star selector mirrors (SSM1 \& SSM2) and an ad hoc model of flexure rates as a function of sky position. We therefore levy the looser requirement of 20 mas passive stability in $300 \mathrm{~s}$ on the full optical system, budgeted in equal parts to the HOWFS, optical relay, and the optical bench itself.

The allowable misalignment in the pupil plane scales with the subaperture size, and thus becomes very small in extreme $\mathrm{AO}$ systems. We require that wavefront sensor microlens arrays remain aligned to DM actuators to $5 \%$ of the finest subaperture over a $300 \mathrm{~s}$ integration, which corresponds to $90 \mu \mathrm{m}$ at the mirrors and $8 \mu \mathrm{m}$ at the microlens arrays. All mechanical elements have been evaluated with finite element analysis and stiffened as necessary to meet the flexure requirements.

\section{DEFORMABLE MIRRORS}

High spatial frequency wavefront control will be provided by a Xinetics Inc. photonex module deformable mirror with 3388 active actuators on a $66 \times 66$ grid with $1.8 \mathrm{~mm}$ pitch (Figure 2). Actuator stroke was measured to be $1.8 \mu \mathrm{m}$ before bonding of the face sheet and $\sim 1.2 \mu \mathrm{m}$ in the final assembled mirror, limited to a linear range by the high voltage amplifiers. All actuators are functional with an RMS gain uniformity of $9 \%$. The mirror facesheet is currently undergoing final polishing meet our required maximum unpowered surface figure of $200 \mathrm{~nm}$ peak-to-valley. 
A second "woofer" DM provides the additional stoke required to compensate atmospheric turbulence across the 5.1 $\mathrm{m}$ aperture. We are reusing the PALMAO DM for this purpose, which has 241 active actuators on a $7.0 \mathrm{~mm}$ pitch and 4 $\mu \mathrm{m}$ of stroke. The tweeter DM is located at the pupil with an angle of incidence of $10.5^{\circ}$, while the woofer is at a conjugate height of $+792 \mathrm{~m}$ and $16.0^{\circ}$ incidence. The woofer conjugate height has been minimized to reduce focal plane distortions caused by compensating low-order modes at a non-pupil conjugate. Space constraints prohibited us from including a second relay.

Electronics and cabling for the DM3388 have proven to be one of the more costly implementation issues. The two full-height racks of high-voltage drivers dissipate $4 \mathrm{~kW}$, and cable volume constraints have forced us to locate these at the Cassegrain focus, requiring liquid cooling systems to be installed. The cables running from drivers to mirror have themselves required a redesign to facilitate making the 4752 necessary electrical connections (4356 actuator signals and 396 bias voltages) in a reasonable amount of time during daytime installation of the instrument.

\section{WAVEFRONT SENSOR}

PALM-3000's deformable mirrors will be driven by signals from a Shack-Hartmann wavefront sensor which supports both adjustable pupil sampling and frame rates. Selection of the pupil sampling is achieved with a microlens array exchange mechanism which can select from four different microlens arrays that span 8, 16, 32 and 63 subapertures across the re-imaged pupil. Control over the spatial and temporal pupil sampling serves to minimize total wavefront reconstruction error over a wide range of guide star brightness ${ }^{17}$, which in turn maximizes the delivered Strehl ratio. The sensor also incorporates a tilted spherical mirror and cylindrical lens to match the $10.5^{\circ}$ angle-of-incidence projection of the telescope pupil on the high-order deformable mirror. The intended registration of subapertures to high-order deformable mirror actuators is presented in Figure 3. In the 8,16 and 32 across sampling modes, the pupil is centered on the $128 \times 128 \mathrm{CCD}$ detector pixels, while in the 63 across mode, the pupil is offset slightly using the optical relay star selection mirrors (SSMs) to enforce a Fried geometry with respect to the high-order deformable mirror actuator. For a full description of the wavefront sensor design, we refer the reader to Baranec $(2008)^{15}$.

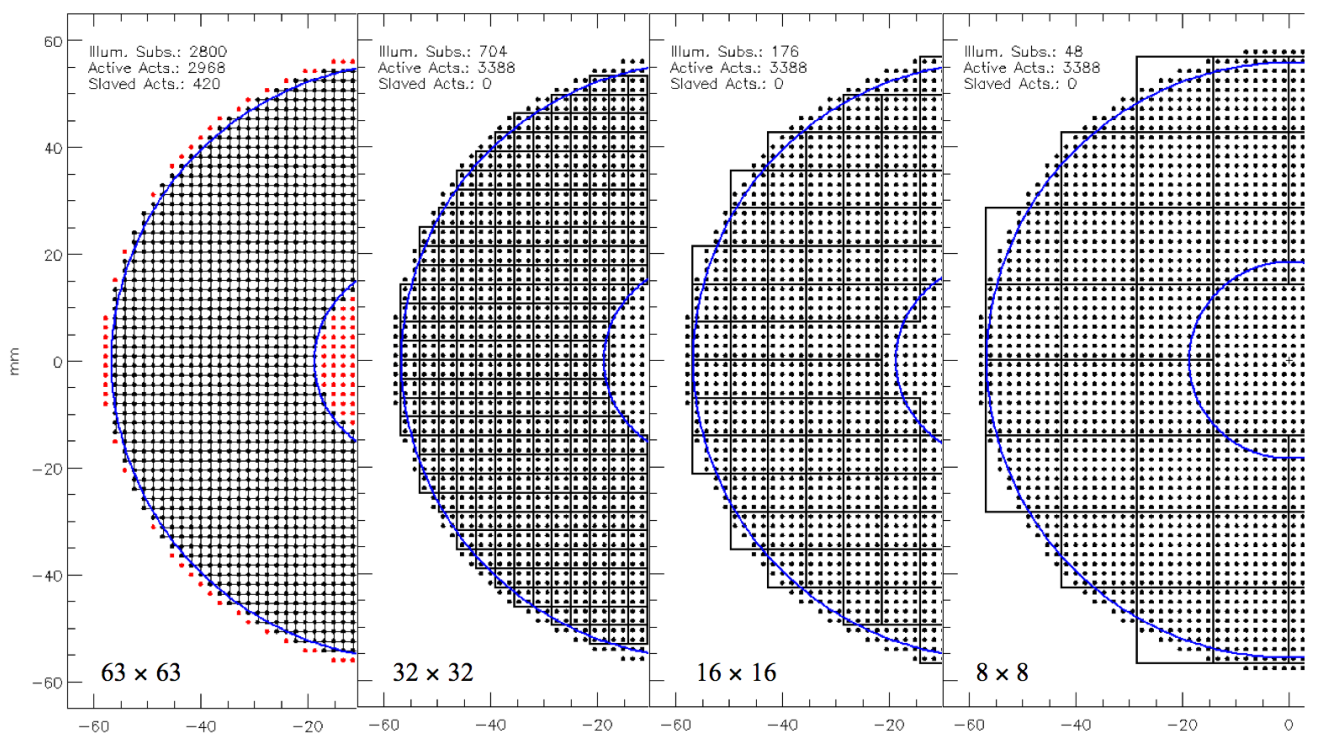

Figure 3. Alignment of high-order deformable mirror actuators to HOWFS subapertures in the 4 operating modes. The Fried geometry between the high-order deformable mirror and wavefront sensor is preserved in the $63 \times 63$ mode.

The wavefront sensor has been assembled and aligned as seen in Figure 4, with preliminary testing currently underway. A telescope simulator (not shown) which mimics the F/\# and pupil location of the PALM-3000 optical relay was used to aid in the alignment of the wavefront sensor. The CCD50 detector is attached to a kinematically repeatable mount and is able to be removed and replaced with a high-resolution CMOS camera with $3.5 \mu \mathrm{m}$ pixels (Pixelink PLB781F-BL) for fine alignment. The CMOS camera is used to register each of the four microlens arrays with the re- 
imaged pupil as well as determine the position of the microlens focus compensation stage which gives the best focus of each of the Shack-Hartmann patterns. The left two panels of Figure 5 present images from the CMOS camera showing microlens array registration of the 8 and 63 across sampling modes, acquired by slightly refocusing images of the pupil. (Note that the shifting of the pupil was not easily achieved without the SSMs feeding the wavefront sensor so 64 subapertures are temporarily illuminated.) Final optical testing is done with the CCD50 reinstalled into the wavefront sensor assembly. The third and fourth panel of Figure 5 shows the resulting image planes as seen by the CCD50 for the 8 and 63 across sampling modes. Table 1 presents the preliminary residual static wavefront errors within the sensor.

With the wavefront sensor aligned and tested, it will next be integrated with the high-order deformable mirror and real-time reconstructor computer in the fall of 2009. Optical registration of the different wavefront sampling modes will be tested with the high-order deformable mirror. In particular, the procedure for matching the Fried geometry with the 63 across wavefront sensing mode will be perfected.

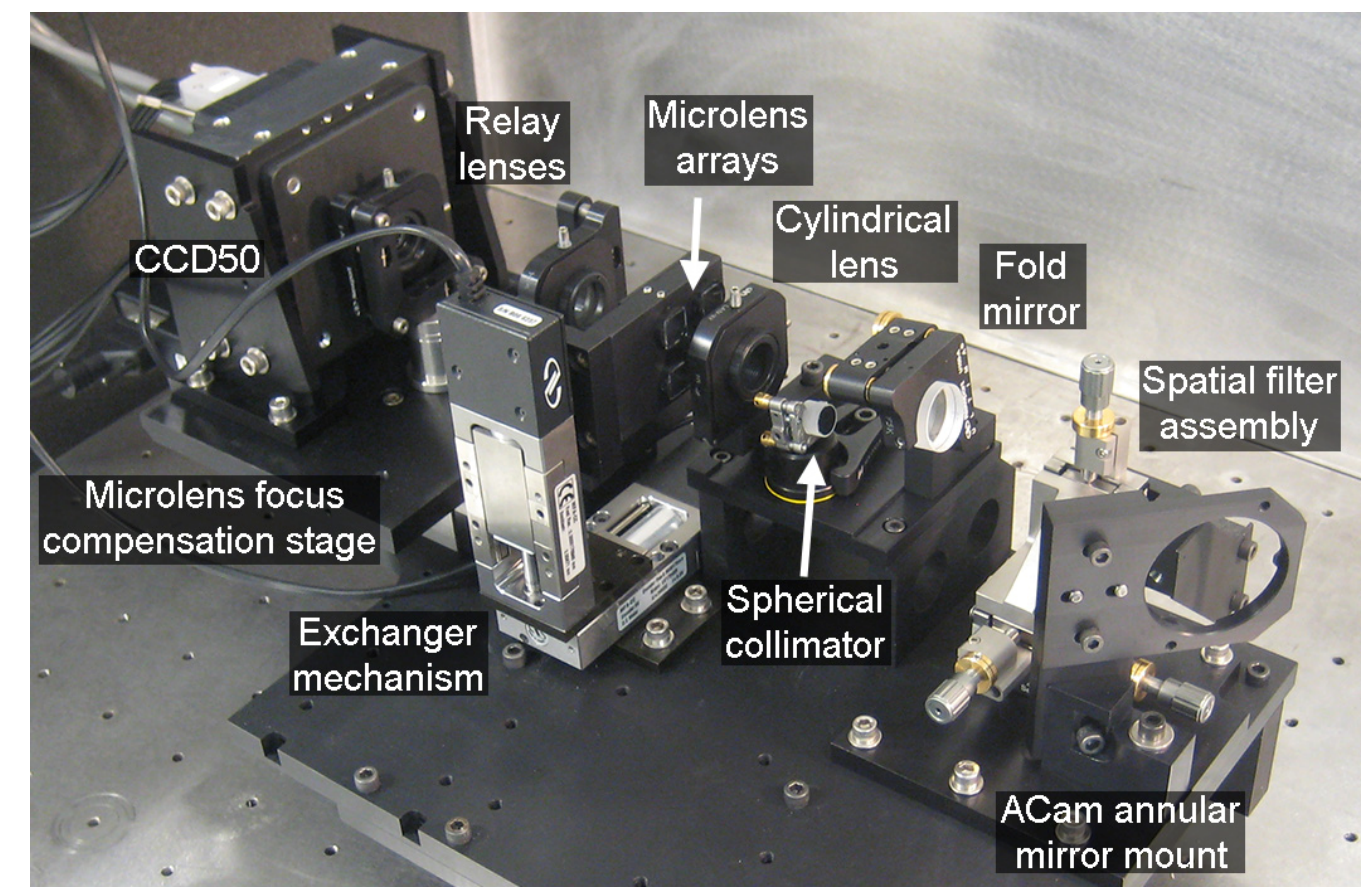

Figure 4. Current wavefront sensor assembly with light baffles removed. The acquisition camera will sit on the left side of the platform, fed by an annular mirror just ahead of the spatial filter assembly. The entire assembly is mouted on a linear focus stage.

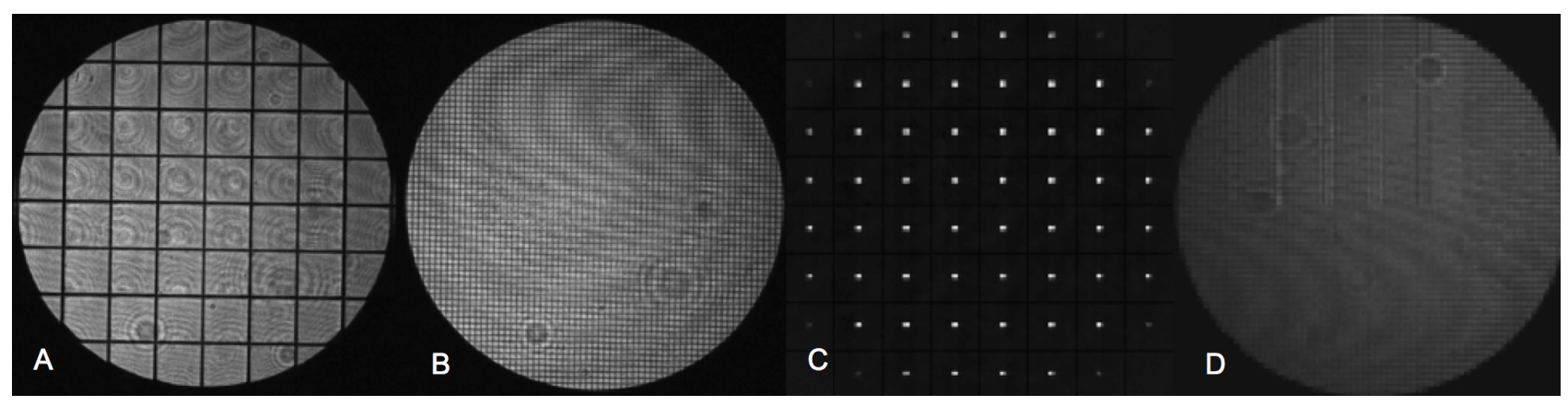

Figure 5. Images of the wavefront sensor pupil and focal plane. (A) and (B) were taken with the CMOS detector through the 8 and 63 across microlens arrays, respectively. (C) and (D) are CCD50 images of the ShackHartmann spot patterns from the 9 and 63 across microlens arrays, respectively. 
Table 1. Preliminary residual static wavefront errors within the wavefront sensor.

\begin{tabular}{ccc}
\hline $\begin{array}{c}\text { Pupil spatial sampling } \\
\text { (subapertures) }\end{array}$ & $\begin{array}{c}\text { RMS slope value } \\
\text { (pixels) }\end{array}$ & $\begin{array}{c}\text { RMS wavefront error } \\
\text { (nm; Zernike polynomials 1-20) }\end{array}$ \\
\hline 63 & 0.038 & 102 \\
32 & 0.066 & 166 \\
16 & 0.150 & 143 \\
8 & 0.175 & 150 \\
\hline
\end{tabular}

\section{WAVEFRONT CONTROL}

We are implementing a flexible servo control architecture to facilitate the testing of several control schemes. Our primary operating mode (mode 1) is illustrated in Figure 6. The reconstructor matrix is computed following the prescription of Conan et al ${ }^{18}$, splitting commands to the two deformable mirrors based on spatial frequency. The $\sim 3000$ high-order spatial modes compensated by the HODM are derived from the influence functions of the illuminated HODM actuators. The remaining 200 low-order modes controlled by the LODM (including tip/tilt) are computed to be orthogonal to the HODM modes. Offload loops operating with a one-frame delay filter blind modes from both deformable mirrors' commands, shift any spurious low-order modes from the HODM to the LODM, and offload tip/tilt from the LODM to the TTM. The two-stage tip/tilt control (first with the LODM, then offload to the TTM) allows us to benefit from the higher control bandwidth of the LODM with respect to our rather sluggish monolithic TTM. The shape each mirror assumes in the absence of an error signal is determined by a "flatmap", added with low gain to the error signal.

During operation with the Project 1640 instrument, the post-coronagraphic calibration wavefront sensor will provide high spatial frequency "truth" measurements, approximately once per minute. This error signal will be decomposed into HOWFS-blind and HOWFS-visible components. HOWFS-visible components will be added to the centroid offsets to which the system is driven, while HOWFS-blind components will be incorporated into the HODM flatmap.

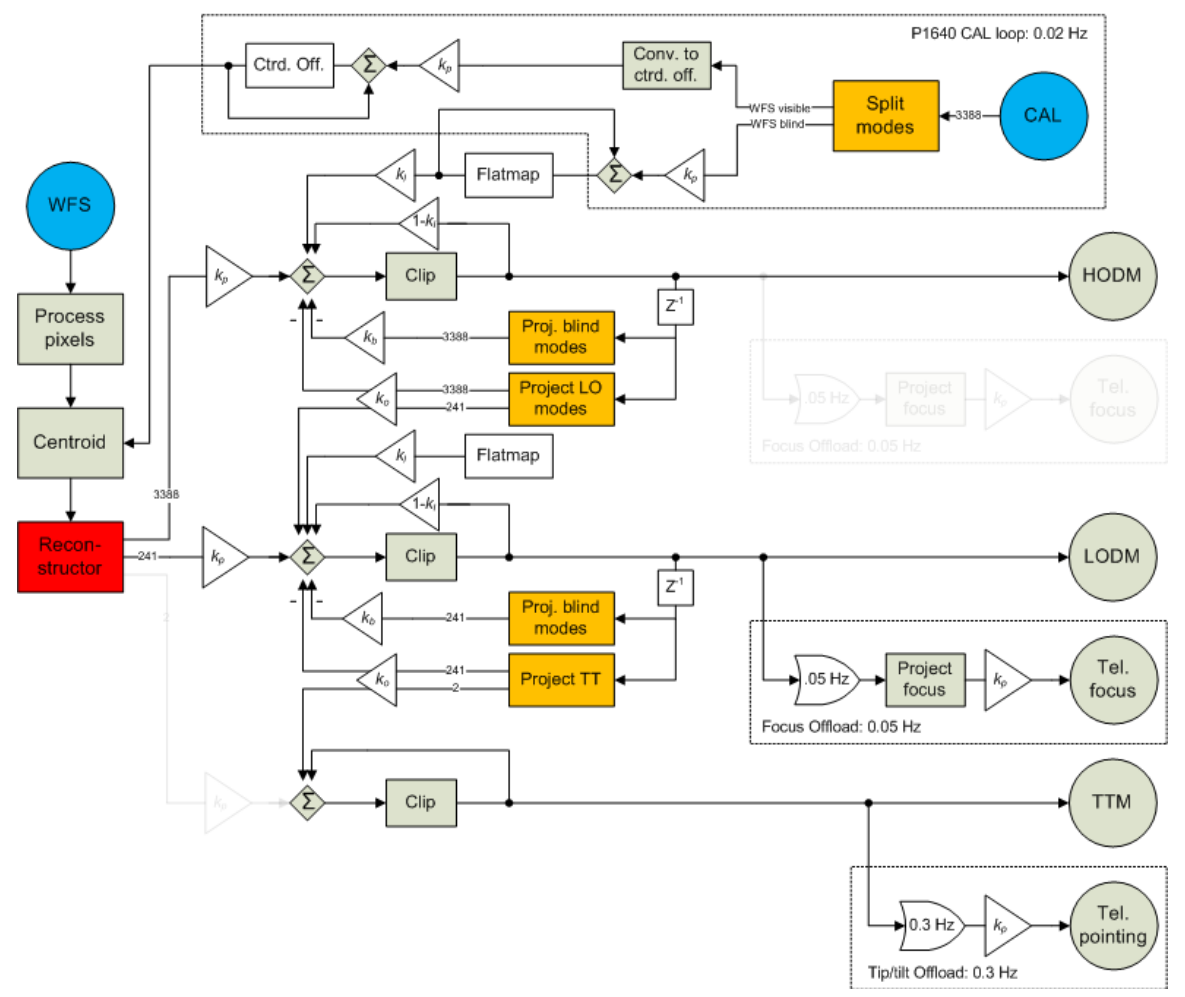

Figure 6. Block diagram for servo control mode 1. Control signals to the LODM and HODM are split by spatial frequency. The Project 1640 calibration wavefront sensor updates the HOWFS centroid offsets and the shape to which the HODM is driven in the absence of an error signal. 
An alternative servo control mode whose performance we intend to compare on sky (mode 2) will divide the HOWFS error signal only between the HODM and TTM. With a one frame delay, low-order modes detected on the HODM will be offloaded to the LODM. While having lower bandwidth for low-order modes, this scheme eliminates the possibility of the DMs fighting one another by assuming equal and opposite surface figures, and allows a simple zonal reconstructor to be used.

The PALM-3000 wavefront processor computer has been specified to perform full vector matrix multiplication reconstruction of the guidestar wavefront at 63 across sampling and $2000 \mathrm{~Hz}$ frame rate ${ }^{16}$. With a total of 3631 actuators to control and 8192 slope measurements (though some fall outside the illuminated pupil, they are included in the calculation for symmetry), the computational problem is over 2 orders of magnitude larger than that for the most complex current astronomical AO systems. Our novel solution uses 17 NVIDIA model 8800 graphics processor units (GPUs) hosted in a cluster of 9 desktop personal computers running a real-time Linux operating system, all located in a computer room remote to the instrument. Using entirely off-the-shelf hardware, a GPU cluster provides the greatest ease of use, potential upgradeability, and cost savings. By dividing the primary wavefront reconstruction computation between 16 GPUs and further processing the HOWFS subapertures in two sequential batches (beginning when just half the pixels have been read out) a total computational latency of only $222 \mu \mathrm{s}$ is achieved. Offload commands between adaptive mirrors will be computed on the $17^{\text {th }}$ GPU. We expect that the latency can be further reduced by future upgrades of the GPUs, which enjoy a rapid development cycle due to strong commercial demand.

\section{LABORATORY TESTBED}

A laboratory testbed for PALM-3000 is being integrated at Caltech in summer 2009. Its purpose is to test all major new components and interfaces of the PALM-3000 system prior to decommissioning the currently operational PALMAO system. Using a refractive relay in place of the off-axis parabolas of the final system, a monochromatic light source will be collimated, reflected off the HODM at $10.5^{\circ}$ incidence angle, and refocused. Three output foci will be provided using pellicle beamsplitters: the PALM-3000 HOWFS, a scoring camera, and an Imagine Optic HASO calibration wavefront sensor. A phase-shifting interferometer will simultaneously measure the HODM surface figure at $0^{\circ}$ incidence angle. The full complement of electronics and cabling will be used to control the HOWFS and HODM, while the wavefront processor computer cluster is housed in the laboratory next door.

Subsystems will be integrated into the testbed as they are completed, beginning with the HOWFS in September 2009. This simple testbed will allow us to validate the performance of the HODM, HOWFS, and wavefront processor computer. It also provides a platform for measuring HODM influence functions, and computing and testing reconstructor matrices. Finally, the electronic and software interfaces between all major components will be thoroughly tested, significantly reducing risk during the final integration phase.

\section{CONCLUSIONS}

With 3388 active actuators, PALM-3000 will be the highest order adaptive optics system ever deployed for astronomy. It will deliver high Strehl in the near-infrared ( $88 \%$ in $\mathrm{H}$ band) and diffraction-limited correction throughout the visible to a suite of instruments optimized for low wavefront error science. Using bright natural guide stars, the Project 1640 coronagraphic IFU should achieve contrast levels of better than $10^{6}: 1$ at $0.5 \%$, facilitating the direct imaging and spectroscopic study of Jovian planets around nearby young stars. Many challenges remain in the final integration and deployment of PALM-3000, but with a 100\% functional high-order deformable mirror and most other subsystems meeting their design specifications, we are confident that the instrument will meet its scientific goals. We expect the testbed phase to conclude by June 2010, to be followed by final integration with the PALMAO components in fall 2010, and PALM-3000 first light in February 2011.

\section{ACKNOWLEDGMENTS}

This research was supported by National Science Foundation grant AST-0619922, PI: Dr. R. Dekany. Support for the wavefront reconstructor computer has been provided by the Air Force Office of Sponsored Research, Award \#FA955006-1-0343 (DURIP FY06). Support for the PALM-3000 3388 actuator DM is provided by a NASA Small Business Innovation Research grant \#NNG06CA21C. Generous support for the adaptive optics interface to the SWIFT instrument has been provided by Ron and Glo Helin. 


\section{REFERENCES}

1. T. Nakajima et al., "Discovery of a cool brown dwarf", Nature 378, 463-465 (1995).

2. R. Neuhauser et al., "Evidence for a co-moving sub-stellar companion to GQ Lup", Astronomy \& Astroph. 435, L13 (2005).

3. G. Chauvin et al., "Giant planet companion to 2MASSW J1207334-393254", Astronomy \& Astroph. 438, L29 (2005).

4. C. Marois et al., "Direct imaging of multiple planets orbiting the star HR 8799", Science 322, 1348-1352 (2008).

5. S. Hinkley et al., "A new integral field spectrograph for exoplanetary science at Palomar", Proc. SPIE 7015, 701519 (2008).

6. S. Hinkley et al., "Temporal evolution of coronagraphic dynamic range and constraints on companions to Vega", Astroph. J. 654, 633-640 (2007).

7. B.A. Macintosh et al., "The Gemini planet imager: from science to design to construction", Proc. SPIE 7015, 701518 (2008)

8. J.-L. Beuzit et al., "SPHERE: a planet finder instrument for the VLT", Proc. SPIE 7014, 701418 (2008).

9. Oppenheimer, B. R., Dekany, R. G., Troy, M., Hayward, T. L., Brandl, B., "Companion detection limits with adaptive optics coronagraph," Proc. SPIE, 4007, 899 (2000).

10. T. L. Hayward et al., "PHARO: A Near-Infrared Camera for the Palomar Adaptive Optics System," PASP 113, 105-118 (2001).

11. E. Serabyn et al., "Extreme adaptive optics imaging with a clear and well-corrected off-axis telescope subaperture", Astroph. J. 658, 1386-1391 (2007).

12. N. Thatte et al., "The Oxford SWIFT integral field spectrograph", Proc. SPIE 6269, 62693L (2006).

13. M. Troy et al., "Palomar Adaptive Optics Project: Status and Performance", Proc. SPIE 4007, 31-40 (2000).

14. A. H. Bouchez et al., "The PALM-3000 high order adaptive optics system for Palomar Observatory", Proc. SPIE 7015, 70150Z (2008).

15. C. Baranec, "High Order Wavefront Sensing System for PALM-3000", Proc. SPIE 7015, 70155M (2008).

16. T. Truong et al., "Real-time Wavefront Control for the PALM-3000 High Order Adaptive Optics System", Proc. SPIE 7015, 70153I (2008).

17. C. Baranec and R. Dekany, "Study of a MEMS-based Shack-Hartmann wavefront sensor with adjustable pupil sampling for astronomical adaptive optics," Applied Optics 47, 5155-5162 (2008).

18. R. Conan et al., "Distributed modal command for a two-deformable-mirror adaptive optics system", Applied Optics 46, 4329-4340 (2007). 